\title{
MOLECULAR CLONING OF cDNA ENCODING HUMAN LANOSTEROL SYNTHASE
}

\author{
Chung Ki SUNG, ${ }^{1)}$ Masaaki SHIBUYA, Ushio SANKAWA ${ }^{2)}$ and Yutaka EBIZUKA*
}

Faculty of Pharmaceutical Sciences, The University of Tokyo, 7-3-1 Hongo, Bunkyo-ku, Tokyo 113, Japan

A cDNA encoding human lanosterol synthase, the enzyme responsible for the backbone formation step in sterol biosynthesis, was cloned by extensive application of PCRs. Five degenerate oligonucleotide primers $(139 \mathrm{~S}, 440 \mathrm{~S}, 528 \mathrm{~A}, 575 \mathrm{~A}$ and $712 \mathrm{~A})$ corresponding to the homologous amino acid sequences among the known 2,3-oxidosqualene cyclase(OSC) were designed. PCR with one pair(440S and 528A) of five primers yielded a 285-bp fragment. PCRs with the primers based on the obtained fragment and the degenerate primers (139S and 712A) gave longer fragments. Finally, full nucleotide sequence of cDNA was obtained by a "rapid amplification of cDNA ends" (RACE) method.

KEY WORDS lanosterol synthase; cDNA; human liver; PCR; 2,3-oxidosqualene; lanosterol; cyclase

Lanosterol synthase catalyzes the cyclization of 2,3-oxidosqualene to lanosterol, which is one of the most complex biosynthetic reactions found in nature ${ }^{3)}$ and is situated in the center of cholesterol biosynthesis of mammals. Control of cholesterol biosynthesis at a later stage, after HMG-CoA reductase, is preferable for development of cholesterollowering drugs since inhibition of HMG-CoA reductase blocks production of dolichol, ubiquinone and protein prenylations. In this context, sterol biosynthetic enzymes such as squalene synthase, squalene epoxidase and lanosterol synthase are promising targets for control of cholesterol biosynthesis. Among these enzymes, lanosterol synthase plays a crucial role in the regulation of intracellular cholesterol level because of its dual functions. ${ }^{4,5)}$

We have purified lanosterol synthase to homogeneous protein from rat liver ${ }^{6)}$ and cloned its cDNA for the first time from a mammalian source by PCR with the primers based on amino acid sequences of the purified protein." Human lanosterol synthase is more attractive than rat lanosterol synthase, since human organs, tissue and enzyme are the ultimate targets for medicinal and pharmaceutical purposes, though it is hard to obtain human liver for purification of enzyme protein. As a part of our continuing studies on steroid and cyclic triterpenoid biosynthesis, we cloned human liver lanosterol synthase gene, taking advantage of the homology found in four known OSCs. Alignment of OSC amino acid sequences of rat, ${ }^{7)}$ Saccharomyces cerevisiae, ${ }^{8,9)}$ Candida albicans ${ }^{10)}$ and Arabidopsis thaliana ${ }^{11)}($ Fig.2) shows that there are plenty of homologous regions, of which the sequences DGGWGLH, KG(A/G)(F/W)PFST, DY(T/P)YVECT, DGSW(E/Y) GSWG, and FPI(W/K)ALG, located at 139-145, 440-447, 528-535, 575-583 and 712-718 in rat lanosterol synthase, were chosen for primer design.

Reverse transcribed (SuperscriptII ${ }^{\mathrm{TM}}, \mathrm{BRL}$ ) cDNA was prepared from human fetal liver poly $\left(\mathrm{A}^{+}\right) \mathrm{RNA}$ (CLONTECH, CL6521-1) and used as a template in the following PCR. Two of the above primers, 139S(5'-GAYGGIGGIT GGGGIYTICA-3') and 440S(5'-AARGGIGCITGGCCITTYWSIAC-3'), were synthesized in the sense direction, and the other three, 528A(5'-GTRCAYTCIACRTAIGGRTAITC-3'), 575A(5'-CCCAISWICCITMCCAISWICCRTC-3') and 712A (5'-CCIARIGCCCADATIGGRAA-3'), were synthesized in the anti-sense direction for corresponding amino acid sequences. Nested PCR, which was effective for the cloning of rat $\mathrm{cDNA}^{7}$, was applied from the beginning. The first PCR product, obtained by using 139 S and $575 \mathrm{~A}$ primers, served as a template of the second PCR. But the second PCR with $440 \mathrm{~S}$ and 528A primers gave no apparent amplified bands of the expected size when analyzed by agarose 


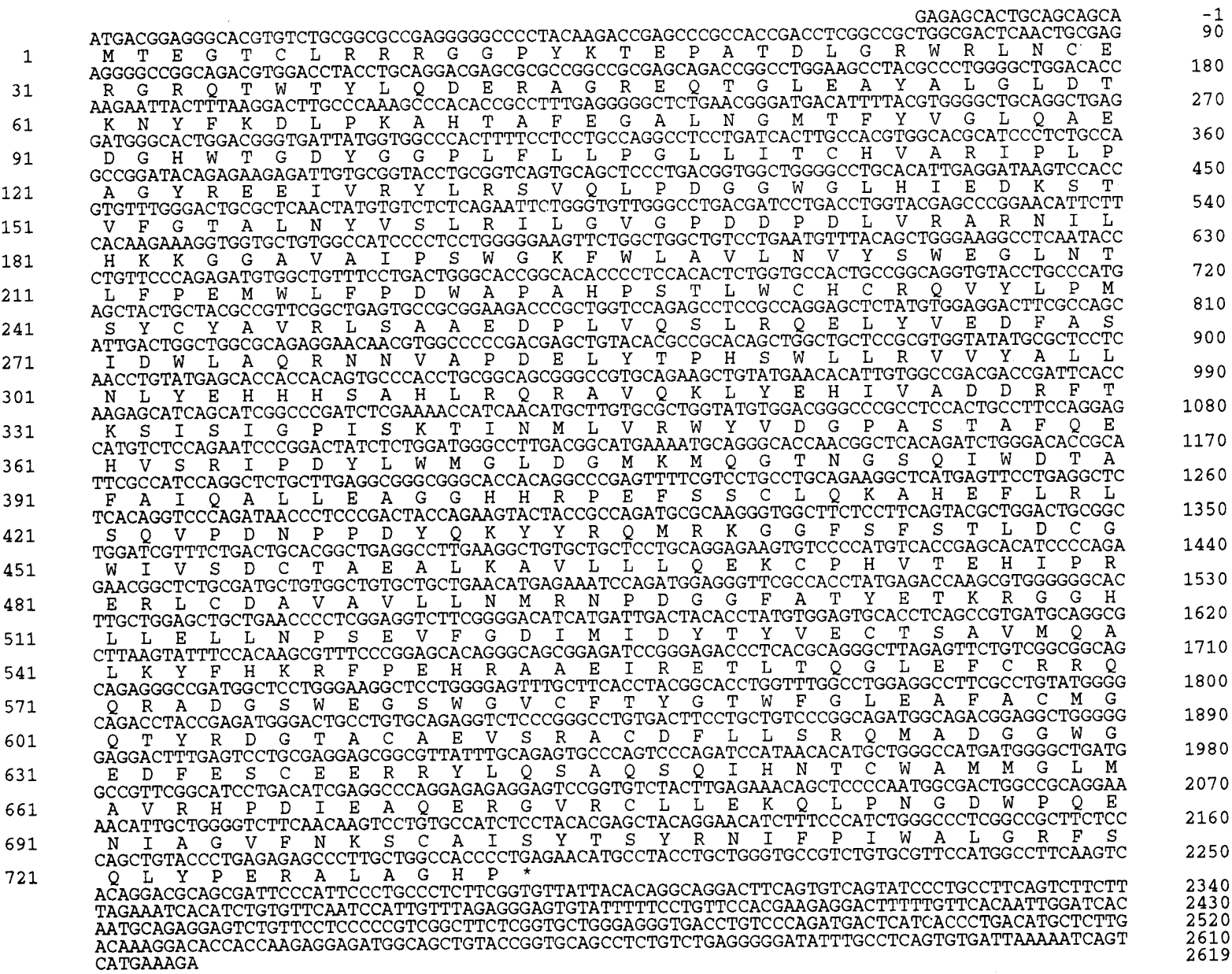

Fig. 1. Nucleotide and Deduced Amino Acid Sequences of Human Liver Lanosterol Synthase cDNA Nucleotides are numbered from the deduced initiation code ATG, and amino acid residues are from corresponding methionine.

electrophoresis. However, the fragment of expected size(285-bp) was obtained when PCR was repeated twice using $440 \mathrm{~S}$ and 528A primers. This fragment was subcloned into pT7Blue $(\mathrm{R})$ and sequenced. The deduced amino acid sequence of this fragment showed $88 \%$ identity to that of rat lanosterol synthase, indicating it to be a part of cDNA of human lanosterol synthase. This fragment was extended toward the 5'- and 3'- ends by PCRs using primers based on the sequence of this 285 -bp fragment, primers $139 \mathrm{~S}$ and $712 \mathrm{~A}$. Finally, a full nucleotide sequence of cDNA was obtained by application of RACE method. ${ }^{12)}$ As shown in Fig. 1, the full nucleotide sequence revealed the presence of 2196-bp ORF that encodes a 732 amino acid polypeptide with a molecular mass of $83 \mathrm{kDa}{ }^{13)}$ Based on the amino acid sequences of affinity-labeled peptide of rat lanosterol synthase, a possible catalytic function of the motif DDXX(D/E) corresponding to DCTAE around C-457 in rat lanosterol synthase has been proposed. ${ }^{14)}$ However, our previous finding in rat $\mathrm{CDNA}^{7)}$ and our present finding in human cDNA that there are no $\operatorname{DDXX}(\mathrm{D} / \mathrm{E})$ indicate that some experimental mistakes occurred in their peptide sequencing. The deduced amino acid sequence of the human lanosterol synthase exhibits $83 \%$, $40 \%, 38 \%$ and $42 \%$ identity to those of the lanosterol synthases of rat, ${ }^{7)}$ S. cerevisiae, ${ }^{8,9)}$ and C. albicans ${ }^{10)}$ and that of 2,3-oxidosqualene:cycloartenol cyclase of $A$. thaliana,${ }^{11)}$ respectively. Only one gap between the positions 40 and 41 in the human lanosterol synthase was required to make the maximum matching to rat enzyme. The role of this gap is obscure but will be clarified by molecular biological studies such as site-directed mutagenesis. The expression of this cDNA in erg7 deficient yeast mutant is now in progress to obtain human enzyme protein in quantities for mechanistic studies and screenings for its specific inhibitor from natural products. 


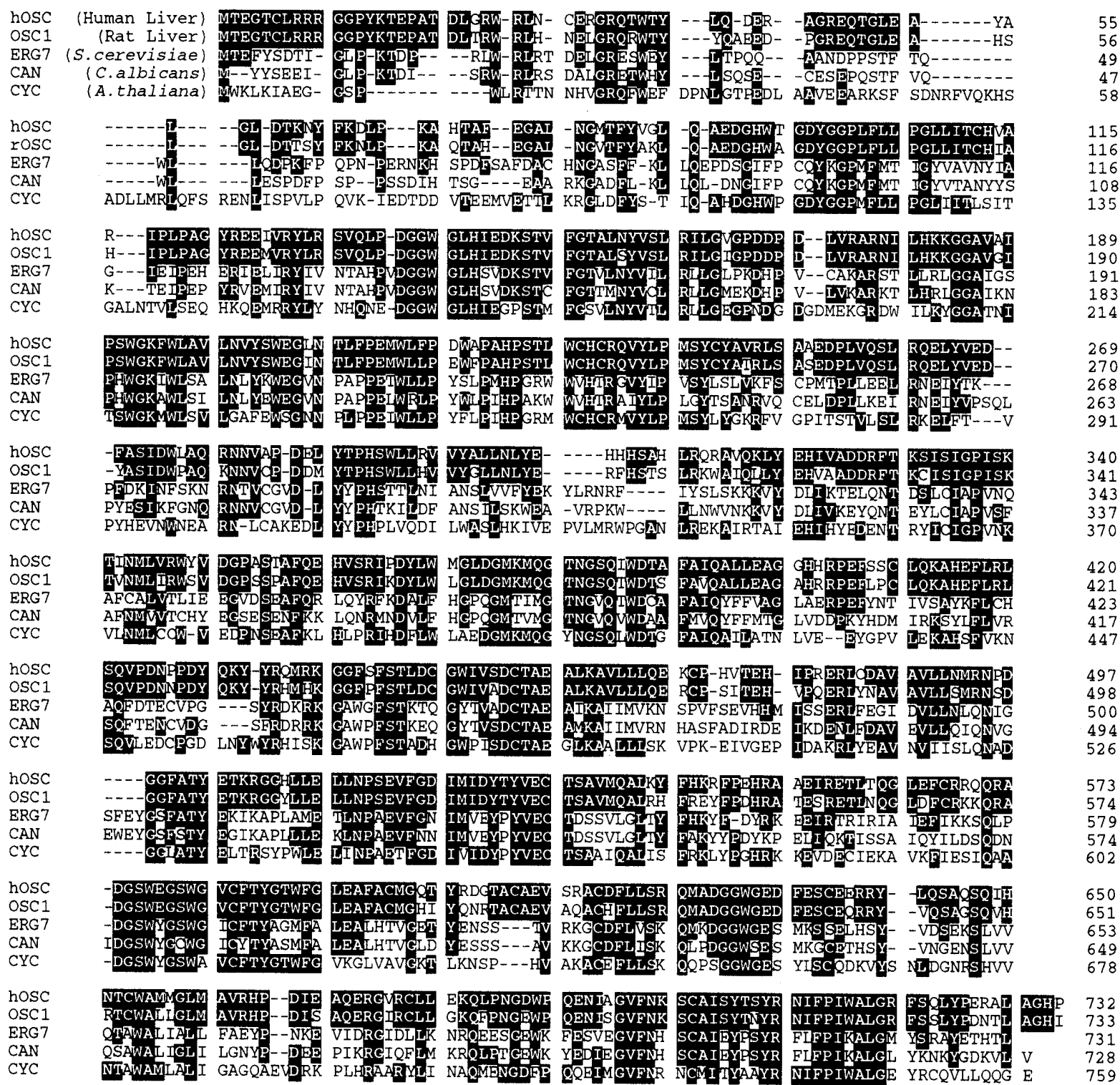

Fig. 2. Comparison of Amino Acid Sequence of Human Liver Lanosterol Synthase to Other OSCs The deduced amino acid sequences of lanosterol synthase of rat $(\mathrm{OSC1}), S$. cerevisiae (ERG7) and $C$. albicans (CAN) are from Refs. 7, 8 and 10, and that of 2,3-oxidosqualene: cycloartenol cyclase of $A$. thaliana (CYC) is from Ref. 11. Hyphens were inserted to maximize homology. Amino acid residues identical to that of human liver lanosterol synthase are boxed.

REFERENCES AND NOTES

1) Present address: College of Pharmacy, Chonnam National University, 300 Yongbong-dong, Buk-ku, Kwangju 500-757, Korea.

2) Present address: Faculty of Pharmaceutical Sciences, Toyama Medical \& Pharmaceutical University, 2630 Sugitani, Toyama-shi, 930-01, Japan.

3) Abe I. , Rohmer M., Prestwich G. D. , Chem. Rev., 93, 2189 (1993) and references cited therein.

4) Spencer T.A., Acc. Chem. Res., 27, 83 (1994).

5) Boutaud O., Dolis D., Schuber F., Biochem. Biophys. Res. Commun., 188, 898 (1992).

6) Kusano M., Abe I., Sankawa U., Ebizuka Y., Chem. Pharm. Bull., 39, 239 (1991).

7) Kusano M., Shibuya M., Sankawa U. Ebizuka Y., Biol. Pharm. Bull., 18, 195 (1995).

8) Corey E. J., Matsuda S. P. T., Bartel B., Proc. Natl. Acad. Sci. USA, 91, 2211 (1994).

9) Shi Z., Buntel C. J., Griffin J. H., Proc. Natl. Acad. Sci. USA, 91, 7370 (1994).

10) Buntel C. J., Griffin J. H., J. Am. Chem. Soc., 114, 9711 (1992).

11) Corey E. J., Matsuda S. P. T., Bartel B., Proc. Natl. Acad. Sci. USA, 90, 11628 (1993).

12) Frohman M A., Dush M. K., Martin G. R., Proc. Natl. Acad. Sci. USA, 85, 8998 (1988).

13) The nucleotide sequence of $h O S C$ will appear in the DDBJ, EMBL and GenBank nucleotide sequence databases under the accession number D63807. The sequence of coding region is $>99 \%$ identical to that recently reported by Baker et al. (Baker, C.H., Matsuda, S.P.T., Liu. D.R., Corey, E.J., Biochem. Biophys. Res. Commun. 213, 154 (1995); GenBank accession number U22526), who isolated the human $h O S C$ gene by hybridization methods.

14) Abe I., Prestwich G. D. , J. Biol. Chem., 269, 802 (1994). 